Frick, R., Bich, L. \& Moreno, A. (2019). An organisational approach to biological communication. Acta Biotheoretica. Doi: 10.1007/s10441-019-09342-2.

\title{
An organisational approach to biological communication.
}

\author{
Ramiro Frick \\ Department of Sociology, Universidad Diego Portales, Manuel Rodríguez Sur 415, Santiago, Chile. \\ Department of Philosophy. Universidad Alberto Hurtado, Alameda 1869, Santiago, Chile. \\ Instituto de Filosofía y Ciencias de la Complejidad, IFICC, Los Alerces 3024, Santiago, Chile. \\ Leonardo Bich \\ IAS-Research Centre for Life, Mind and Society. Department of Logic and Philosophy of Science. University \\ of the Basque Country (UPV/EHU). Avenida de Tolosa 70, Donostia-San Sebastian, 20018, Spain. \\ Email: leonardo.bich@ehu.es \\ Orchid number: 0000-0002-2416-112X
}

\section{Alvaro Moreno}

IAS-Research Centre for Life, Mind and Society. Department of Logic and Philosophy of Science. University of the Basque Country (UPV/EHU). Avenida de Tolosa 70, Donostia-San Sebastian, 20018, Spain.

\begin{abstract}
This paper aims to provide a philosophical and theoretical account of biological communication grounded in the notion of organisation. The organisational approach characterises living systems as organised in such a way that they are capable to self-produce and self-maintain while in constant interaction with the environment. To apply this theoretical framework to the study of biological communication, we focus on a specific approach, based on the notion of influence, according to which communication takes place when a signal emitted by a sender triggers a change in the behaviour of the receiver that is functional for the sender itself. We critically analyse the current formulations of this account, that interpret what is functional for the sender in terms of evolutionary adaptations. Specifically, the adoption of this etiological functional framework may lead to the exclusion of several phenomena usually studied as instances of communication, and possibly even of entire fields of investigation such as synthetic biology. As an alternative, we reframe the influence approach in organisational terms, characterising functions in terms of contributions to the current organisation of a biological system. We develop a theoretical account of biological communication in which communicative functions are distinguished from other types of biological functions described by the organisational account (e.g. metabolic, ecological, etc.). The resulting organisational-influence approach allows to carry out causal analyses of current instances of phenomena of communication, without the need to provide etiological explanations. In such a way it makes it possible to understand in terms of communication those phenomena which realise interactive patterns typical of signalling interactions - and are usually studied as such in scientific practice - despite not being the result of evolutionary adaptations. Moreover, this approach provides
\end{abstract}


operational tools to design and study communicative interactions in experimental fields such as synthetic biology.

Keywords: organisation, influence, biological functions, signals, synthetic biology, regulation.

\section{Introduction}

A variety of biological interactions, taking place at different levels of biological organisation (unicellular, multicellular, ecological), are standardly studied and described by scientists in terms of communication or signalling phenomena. The types of organisms and interactions involved in biological communication are highly diversified. Humans can communicate with words and by other means, vervet monkeys emit specific alarm calls in response to certain kinds of predators (Seyfarth et al., 1980a; 1980b), and crabs communicate by performing threat displays when they are competing for resources (Cameron, 1966). However, communication is not restricted to animals. When damaged by herbivores, some plants release volatiles that are detectable by neighbour plants, which in turn activate preventive defence mechanisms against the herbivores (Heil \& Karban, 2010). Bacteria too exhibit communication, for example by means of quorum sensing mechanisms (Waters \& Bassler, 2005). The concept of communication is also used to account for some of the interactions between organisms belonging to different species, such as the case of human hosts and their gut microbiotas (Keely, 2017) and, in Synthetic Biology, to design protocells capable to trigger signalling response in living cells (Gardner et al. 2009; Rampioni et al. 2014; Lentini et al. 2017).

As pointed out by Thom Scott-Phillips, "on first appearances, these phenomena have little in common. Yet they must presumably share something, if we are happy to identify them all as instances of communication" (Scott-Phillips 2009: 245). This paper aims to address this point, by contributing to the investigation of the common theoretical elements that allow to account for all these phenomena in terms of communication.

What is generally meant by 'communication' in Biology? This question is known to be difficult, and there is no agreement among scientists and philosophers alike on a common general framework to address it. The two most widely established accounts available are the 'information' and 'influence' approaches. The traditional and most popular of the two is the information-based approach, according to which communication can be defined in terms of information transfer from a sender to a receiver by means of a signal. The competing account, proposed as an alternative to the information approach, is based on the notion of influence. According to it, communication can be defined in terms of adaptive influence which a sender exerts on a receiver by means of a signal ${ }^{1}$. In this context, as we

\footnotetext{
${ }^{1}$ Because of the historical association of the terms "sender" and "receiver" with Information Theory and the informational vocabulary, some of the advocates of influence approach have proposed to replace them with
} 
will explain in more detail in the following sections, 'influence' is always defined in terms of functional contribution to the sender, and justified in terms of evolutionary adaptations. Currently, the debate on biological communication remains open, with scientists and philosophers divided between the two approaches ${ }^{2}$. Both accounts exhibit several conceptual issues and practical weaknesses that often make it problematic to apply them in scientific research, and which may lead to inconsistencies such as the exclusion of several phenomena usually studied by biologists as instances of communication. ${ }^{3}$

This paper aims to provide an account of communication based on the notion of organisation, and grounded on the theoretical work on biological autonomy, which characterises living systems as self-maintaining systems capable to produce and replace their components through the continuous interaction with their environment (Piaget, 1967; Varela, 1979; Rosen, 1991; Kauffman, 2000; Moreno \& Mossio, 2015). To do so, it focuses specifically on the influence approach, which aims to provide an operational understanding of those phenomena to which scientists refer as 'communication', by characterising them in functional terms. We discuss the strengths and weaknesses of the influence approach, which suffers from some serious conceptual and practical problems, mostly unnoticed and not sufficiently discussed to this day. More specifically, three main issues seem to undermine this approach in its current formulation: (a) a mismatch between theory and scientific practice in the study of biological communication; (b) the conflation of the description of a phenomenon with the explanation of its origin; and (c) the exclusion of the possibility of realising artificial (biosynthetic) models of biological communication through synthetic biology.

We argue that these issues derive from the way in which the notion of functional influence exerted by a sender upon a receiver is currently understood in the field, i.e. in etiological terms as an adaptation. After presenting and discussing these issues in general terms, we examine a case study from communication in rodents, which despite being

other terms: "signaler" and "perceiver" (Owren et al., 2010), "manager" and "assessor" (Owings and Morton, 1998; Morton 2017), "manipulator" and "mind-reader" (Krebs \& Dawkins, 1984), etc. Here we maintain the more usual terms "sender" and "receiver" without implying informational constructs, but just to denote roles within a minimal communication system.

${ }^{2}$ Proponents of informational approach include, among others, Lewis (1969), Otte (1974), Seyfarth et al., 1980a; 1980b; 2010), Hauser (1996), Bradbury and Vehrencamp (2011), Stegmann (2009), Martinez and Godfrey-Smith (2016). Proponents of the influence approach include Dawkins and Krebs (1978), Owings and Morton (1998), Rendall, Owren and collaborators (Rendall et al., 2009; Owren et al., 2010; Rendall \& Owren 2013) and Morton (2017). Proponents of a hybrid information-mediated influence include for example, Scarantino (2010, 2013). See also Godfrey-Smith (2013).

${ }^{3}$ For example, although the characterisation of communication in terms of information is widespread, the very concept of "information"” is controversial. It has proven to be a source of deep disagreement and potential problems, insofar as it relies on demanding theoretical assumptions on the nature of information and of related notions such as representations, that might make this approach difficult to apply to specific cases (see for example Dawkins \& Krebs, 1978; Owren et al., 2010; Kalkman, 2017; Morton, 2017). In this paper we will not go here into the details of the issues related to the use of the concept of information, as we will focus on the influence account instead. 
considered as a typical example of communicative interaction, cannot be accounted for in terms of evolutionary adaptations. We propose a possible solution to these problems, by adopting an organisational framework (Moreno \& Mossio, 2015), which implies a whole rethinking of the notion of functional influence central to this approach to communication: from an etiological account grounded in evolution to an organisational one focused on current systems. We argue that, by adopting an organisational approach to functions (Mossio et al. 2009), the notion of biological communication as influence can be given a theoretical and philosophical justification by defining 'influence' as contribution to the current organisation of the sender. On this conceptual basis, we build a theoretical account of biological communication in which communicative functions are distinguished from other types of biological functions described by the organisational account (e.g. metabolic, ecological, etc.). The resulting organisational-influence approach to communication exhibits the same virtues of the influence approach based on etiological functions, while avoiding its weaknesses. It can be applied to a wider spectrum of communicative interactions, including minimal life and biosynthetic systems, and it does not exclude cases that, while considered as instances of communication in scientific practice, cannot be accounted for in terms of etiological functions. Finally, we respond to some potential objections to the new approach.

\section{The influence approach to communication: influence as an etiological functional concept}

The origins of the influence approach can be traced back to the paper "Animal signals: information or manipulation?" published in 1978 by Richard Dawkins and John Krebs. They argue against the prevailing view of communication as information transfer, and provide an alternative view based on the concept of 'sensorial influence'. The main point of this approach is that from an evolutionary perspective, communicative behaviour is selected because it contributes to the fitness of its bearer. Thus, they argue, organisms which emit signals (senders) are not selected because they provide information to other organisms (receivers). Rather, senders are selected in virtue of influencing the receivers in ways that are beneficial to themselves. The ultimate role of signals, therefore, is not to provide information to the receivers, but to influence them.

The idea of influence refers to situations in which an animal "makes use of another animal's muscle power" (Dawkins \& Krebs, 1978: 283). In order to exist and to reproduce, animals have to deal with different kinds of animate and inanimate objects from their environment. When dealing with an inert object, an animal can rely only on its own

\footnotetext{
${ }^{4}$ The specific word chosen by Dawkins and Krebs for this form of communication is "manipulation". Yet, to avoid an implicit reference to disadvantageous consequences for the receivers implied by the word "manipulation", contemporary proponents of this approach to communication (e.g. Owren et al., 2010: 772) prefer to talk about 'influence'. Hereinafter we will follow this more recent terminology.
} 
muscles. When dealing with an animate object, instead, in addition to using its own muscles, an animal has also the possibility of make use of the senses and muscles of the animate object, which can be influenced or manipulated. In many situations, sensorial influence can be a far better strategy than brute force alone. Indeed, one of the general properties of communication, according to J. B. S. Haldane, is "the pronounced energetic efficiency of signalling: a small effort put into the signal typically elicits an energetically greater response" (Wilson, 1975: 176), and Morton (2017) holds that "signalling substitutes for, or replaces, behaviour that is energetically costly". For example, let us consider advertising for mates: a bird can save a lot of energy by singing from one spot rather than patrolling around its large territory to find a mate (Morton, 2017). These ideas make sense in a context in which communication is understood, as Dawkins and Krebs proposed, as a kind of sensorial influence, exerted by an organism upon another.

At this point some clarifications are needed. First, in this framework influence (and, thus, communication) is understood as a functional concept, grounded in evolutionary history. Second, the influence approach, in its minimal formulation, is a sender-centred approach according to which the capability to emit signals evolved in virtue of its power to trigger in the receiver a response that is functional for the sender. Third, influence is an operational concept which neither necessarily entails the ascription of mental states (such as desires or beliefs about other organisms) to the organisms involved in communication, nor it is restricted to animals, but can in principle take place between any kind of organisms. These three points are central to understand the virtues and problems of the influence approach, and they will be discussed in more detail in the following subsections.

\subsection{Communication as influence: a functional, evolutionary concept}

A central aspect of this account is that influence is employed as a functional concept. To say that a system A influences another system B by means of the trait $\mathrm{T}$, it is necessary but not sufficient that $\mathrm{T}$ triggers a change of state in B. Additionally, it is necessary that $\mathrm{T}$ has the biological function of triggering such changes of state. Thus, communication is a matter of functional influence.

The notion of function grounds an important distinction in biology of communication, namely, that between signals and cues. Both signals and cues trigger changes of state in biological systems. However, only the formers have the biological function of triggering these changes. For example, conspicuous calls by male tungara frogs attract female frogs, but also attract frog-eating bats. Yet, scientists say that male tungara frogs communicate 
with female frogs, but they do not say that they communicate with predatory bats: they characterise males' calls as signals for females, and just cues to bats ${ }^{5}$.

What does it mean to say that a certain trait, such as emitting a signal, has a certain function? This question constitutes one of the major challenges in philosophy of biology, and several different approaches have been developed. However, one specific account has been embraced by virtually all promoters of the influence approach, namely, the etiological approach to biological functions. It defines the function of a trait in terms of its etiology (i.e. its causal history): the functions of a trait are the effects of past instances of that trait that causally explain its current presence. In its most popular version, the etiological approach appeals to the evolutionary process of natural selection (Millikan, 1989; Neander, 1991; Godfrey-Smith, 1994). It provides an understanding of functions as selected effects, and functional traits as adaptations: traits which have been selected in the evolutionary past due to some specific effects or consequences, that have contributed to the adaptation and survival of the ancestors of the organisms to which the traits belong. An example in the context of signalling studies is Otte's (1974) influential distinction between function which evolved because it fostered survival or reproduction - and incidental effect - a byproduct. Along this line, for example, Owren et al. (2010: 774) argue that "the influencebased definition essentially states that any behavior with an evolved function of influencing another individual qualifies as signaling".

One of the main virtues of the etiological approach is its capacity to unambiguously identify functions among the whole set of effects of a trait and, in particular, to draw a distinction between functions and accidental effects. Thus, the difference between cues and signals can be spelled in evolutionary terms, as Diggle et al. (2007) do in the context of intercellular communication: "a signal is defined as 'any act or structure that alters the behaviour of other organisms [and] which evolved owing to that effect [...]'. This definition distinguishes a signal from a cue where the production of substance $\mathrm{X}$ by cell $\mathrm{A}$ has not evolved owing to its effect on cell B" (Diggle et al., 2007: 1242).

So, we can say that the calls of male tungara frogs are signals for female frogs but are cues for bats, because attracting female frogs (and not attracting predatory bats) was the effect for which these calls were selected in the past, and which led to the present existence of calls.

\footnotetext{
${ }^{5}$ For example, when analysing the case of tungara frogs and predatory bats, Page \& Ryan (2005: 841) explicitly characterise mating calls as cues for bats and signals for female frogs: "Prey-localization cues can also be deliberately produced by the prey, such as sexual advertisement signals" (emphasis added). Similarly, Rhebergen et al. (2015: 1) rely on the same distinction: "[...] multimodal signals can improve the detection, localization and discrimination of the sender's signal relative to simpler unimodal signals, and may sometimes increase the overall signal attractiveness [...]. However, eavesdroppers such as predators and parasites also attend to these displays and may often benefit in similar ways as intended receivers from attending to multimodal cues" (emphasis added).
} 


\subsection{Sender-centred and cooperative versions of the influence approach to communication}

Some of the promoters of the influence approach share a common minimal view of communication as sender-centred, according to which (a) the signal emitted by the sender necessarily evolved owing to the response it elicits in the receiver, but (b) the receiver's response did not necessarily evolve to be triggered by the signal (Dawkins \& Krebs, 1978; Owren et al., 2010). This position does not imply a necessary detrimental effect on receivers, but focuses exclusively on the benefits for the senders.

In contrast with this sender-centred definition of communication, other authors consider that 'true communication' should be functional to both sender and receiver. Along these lines, Maynard-Smith \& Harper (2003) and Scott-Philips (2008) provided a cooperative influence-based definition of communication, called "the 'full' adaptationist definition", that crucially demands that the communicative acts must be adaptive for both parties (Carazo \& Font 2010). Taking into consideration these different interpretations, a general definition of biological communication as influence can be built by combining the following clauses:

The system A communicates with system B by means of a trait $\mathrm{T}$ acting as a signal $\mathrm{S}$, if an only if: $\mathrm{B}$

i) when in presence of a trait $\mathrm{T}$ of the system $\mathrm{A}$, a change of state occurs to a system

ii) the trait $\mathrm{T}$ of the system $\mathrm{A}$ has been selected in virtue of producing a change of state in system B;

iii) the response of system $B$ has been selected to be triggered by traits Ts of systems As;

So, although all the promoters of the influence approach agree on the first two clauses, there is disagreement about the third one. The sender-centred version of the functionalinfluence approach to communication only includes clauses i) and ii), while the cooperative version of the functional-influence approach includes all the three clauses.

Since the following discussion and arguments apply both to the sender-centred and cooperative versions of the influence approach to communication, we do not need to adjudicate between them. For simplicity, however, we will focus on the sender-centred version.

\subsection{Influence neither implies the ascription of mental states, nor is restricted to animals}

When Dawkins and Krebs (1978) define communication, they refer to animals, and their use of the word 'manipulation' can mislead us into thinking that some mental or high cognitive capacities are necessary. However, the concept of sensorial influence in principle neither implies the ascription of mental states, nor it restricts communication to interactions between animals exhibiting high cognitive functions supported by a nervous system. In 
fact, plants can exert sensorial influence. For example, a large number of orchids attracts pollinators by producing structures that mimic visual, olfactory and tactile features of conspecific females of pollinators, thus exploiting the perceptual biases of these animals (Schaefer \& Ruxton, 2009). The orchid Chiloglottis trapeziformis, for example, lures males of the thynnine wasp (Neozeleboria cryptoides) by releasing a compound identical to a sex pheromone normally produced by female thynnine wasps. The males are enticed to mate with the flower, and in the process of attempting to do so, they pick up pollen from the flower. Then, they transfer it to a second plant when lured again by the pheromonemimicking odour (Schiestl et al., 2003). This kind of influence occurs between bacteria as well. Veillonella atypica and Streptococcus gordonii are two species involved in the early colonisation of the human oral cavity. V. atypica requires the presence of $S$. gordonii, because the latter ferments sugars and releases lactic acid, which constitutes the preferred carbon source for the former. In order to be able to colonise dental surfaces, V. atypica produces a soluble chemical signal that triggers amylase expression in $S$. gordonii, thereby increasing the degradation of complex carbohydrates and lactic-acid production. In such a way, V. atypica alters the behaviour of $S$. gordonii for its own benefits, with no direct benefit for the latter (Keller \& Surette, 2006).

\section{The limits of the influence approach}

In this section we identify and discuss three major issues that characterise the influence approach to communication as a framework based on an etiological account of functions. One problem consists in the mismatch between the theoretical framework provided by the influence approach and the study of communication in current scientific practice, specifically in relation to the role and importance of considerations on the evolutionary past. The second problem arises from a conceptual conflation of the description of a phenomenon and the explanation of its origin and maintenance. Aspects of both problems are then illustrated in a case study (Sec. 3.3), in which we review some details of the rodent infant-mother communication system mediated by ultrasonic vocalisations. Finally, the third issue concerns the possibility of modelling minimal communication in synthetic biology by means of protocells or synthetically modified cells, which are not the product of evolution by natural selection. This possibility seems to be ruled out in the current formulation of the influence approach. All these problems are directly dependent on the specific account of functions adopted. However, we argue in Section 4, they can be overcome by grounding the influence approach to communication into a different functional framework: the organisational one.

\subsection{A mismatch between the influence-based approach and scientific practice}

According to the influence approach to communication, to count as a signal a trait must have been selected for its capacity to trigger certain changes of state in other systems. In this view, an interaction between organisms is communicative only in virtue of a history of 
selection of similar patterns in the behaviour of their ancestors. As remarked by Di Paolo (1999: 20-21), it follows from this approach that any complex interaction between organisms, "no matter how ritualised or similar to known cases of communication", cannot be considered to be an instance of communication until its selective history has been advanced. This is exactly the claim made by Diggle and colleagues when promoting the influence approach within the field of bacterial communication: "when we see cell A produce a substance $\mathrm{X}$ that elicits a response in cell $\mathrm{B}$, it is tempting to conclude that the substance produced is a signal [...however] to demonstrate that substance $\mathrm{X}$ is a signal and not a cue, it is necessary to show that it evolved owing to the response it elicits." (Diggle et al., 2007: 1242).

From this standpoint, one should expect that when scientists describe an interaction as communication, they do so on the basis of evolutionary evidence. However, often this is not what happens in scientific practice. From an historical point of view, it is interesting to note that long before Darwin developed his theory of evolution by natural selection, several biological interactions were already identified and described as cases of communication (Laidre \& Johnstone, 2013; Fögen, 2014). Moreover, apart from evolutionary biologists, when current scientists describe certain interactions in terms of communication, they do not necessarily rely on evolutionary evidence. Studies on the evolutionary history of signals and communicative behaviours are not always the conceptual core of research on biological communication, and they are often done only after the trait in question has been already described as a signal. For example, the evolutionary history of the bioluminescent communication in fireflies was revealed only recently (Branham et al., 2001), but the characterisation of the phenomenon in terms of communication had already been developed long time ago (e.g. Lloyd 1966).

The mismatch between what one would expect on the basis of the influence approach, and what actually occurs in scientific practice, can be viewed as the result of imposing too high epistemic standards, resulting from an understanding of signals as adaptations. Evolutionary knowledge is difficult to achieve, and reasonable adaptive hypotheses can be held only after long and thorough investigations. In many cases there is no easy access to the evolutionary past. Therefore, it is difficult, and sometimes impossible, to figure out the adaptive history of a trait (Williams 1966, Gould \& Lewontin 1979). An undesirable consequence of the influence approach to communication, thus, is the difficulty for biologists to figure out whether or not traits are signals, and what interactions count as cases of communication.

\subsection{Conflating an operational characterisation and an origin explanation}

The influence approach tries to characterise communication as it is taking place, by pointing to the way communicative interactions have evolved. Yet, these two aspects are not necessarily one and the same thing. As argued by Di Paolo (1997a; 1997b), by pursuing 
this strategy the influence approach conflates the operational characterisation of the phenomenon of communication as observed in current systems, with a plausible explanation of the origin and maintenance of this phenomenon, i.e., its evolution by natural selection.

Merging these two aspects generates methodological problems. If signals, by definition, have to be considered as products of natural selection, then questions about the possibility of a signalling system emerging through neutral, non-selective evolutionary processes should be excluded as meaningless in this framework. Yet, to explain the evolutionary origin and maintenance of any trait, evolutionary biology requires a procedure that consists in testing the different adaptive hypotheses according to which the trait is an adaptation, against alternative neutral hypotheses, such as drift, genetic constraints, by-products of other features of the organism, etc. (see Lloyd, 2015; Pigliucci \& Kaplan, 2000). So, if this is the norm for the study of all biological traits, why should biological signals be an exception?

Moreover, the definition of signals in terms of natural selection seems to exclude $a$ priori the possibility of distinguishing between questions asking how communication has come to exist, and how communication is taking place in current interactions between organisms - the phenomenon studied as an instance of communication. As such, this theoretical framework might get into contrast with research work in those fields of biology involved in characterising phenomena of communication as they are observed in the present.

Taking into consideration how research work proceeds, and which kind of questions are asked, is an important factor at the moment of discussing and evaluating theoretical issues. Without underestimating the importance and value of evolutionary considerations, investigating communication might also need independent theoretical tools and approaches to operationally analyse and model current communicative interactions as proper cases of communication. As pointed out by Salmon (1998), one can in principle distinguish between (etiological) explanations which tell the story leading to the occurrence of a phenomenon, and constitutive ones, which provide a causal analysis of the phenomenon itself. These two different explanatory focuses - origins of traits and current operational characterisations do not necessarily coincide. That might be the case with biological communication. In fact, one can legitimately ask whether it is really a conceivable possibility for a scientist to characterise an interaction that exhibits a certain causal pattern as a communicative signal even if is not an adaptation but, instead, for example, just an incidental by-product of other features of the organism.

In the next section we will address these concerns by examining in detail a recent scientific controversy about the communicative phenomenon of rodent pups' ultrasonic isolation calls (USVs). 


\subsection{A case study: pup rats' ultrasonic vocalisations}

Pup rats' USVs are interactions that follow patterns of behaviour typical of communication understood as influence - in which the response of the receiver contributes to the viability of the sender - and are regarded as a communicative phenomenon in scientific practice. Yet, the influence approach may not be able to categorise and describe this case as an instance of communication by relying on an etiological account of functions.

Let us consider new-born rats. They are altricial animals with limited locomotor and thermoregulatory abilities. They exhibit a great dependence on the warmth provided by the mother and the littermates in the nest. When a new-born becomes isolated from the nest, it emits USVs around the 40-kHz level. The mother can hear these USVs and respond to them by searching for her pup, then grasping it by the nape of the neck, and returning it to the warmth of the nest.

When considered together, these elements suggest the presence of infant-mother communication. This is the reason why infant rodents' USVs have been standardly used for a long time as a measure for the quantification of communication deficits in mouse models

of certain neuropsychiatric disorders (Scattoni et al., 2009; Silverman et al., 2010). The remarkable fit between the behaviour of the pup (the sender), its physiological needs, and the response of the mother (the receiver), suggests that pups' USVs have the function of eliciting retrieval behaviour in the mother, as required by the influence approach to communication. Thus, according to the etiological approach that characterises functions as selected effects, to say that USVs have the function of eliciting maternal retrieval behaviour means that USVs have been naturally selected in virtue of their capacity to elicit such behaviour. Indeed, this is precisely the way in which this trait was adaptively explained (de Ghett 1978).

However, more recent studies have revealed that things are more complicated than previously thought. By investigating the physiology of vocalisations of Norway rats (Rattus norvegicus), Mark Blumberg and colleagues concluded that USVs are not a pup's adaptation to influence its mother. Instead, USVs are just an incidental acoustic by-product of a physiological process related to thermoregulation (Blumberg \& Sokoloff, 2001; Blumberg, 2009).

Before examining the implications of this idea for the debate on communication, let us review some of its details. Temperature is a critical variable in the production and modulation of USVs in isolated infant rats. An isolated pup begins to emit USVs when it is cold: when tested under temperature conditions similar to that of the nest, isolated pups do not emit or emit considerably less USVs. In case of hypothermia, as the heart muscle gets progressively colder, both the rapidity and the strength of its contractions diminish. At the same time, as the blood gets colder, it becomes thicker, more viscous, and harder to pump 
through the blood vessels. Thus, the now-thick venous blood pools remain in the large peripheral veins rather than returning to the heart, and the pup's ability to deliver oxygenated blood to its body is heavily compromised.

When a rat pup is confronted with this critical situation, influencing the mother's behaviour to return to the warmth of the nest looks like the perfect behavioural solution to restore body temperature before the physiological capability of thermoregulation is developed (De Ghett, 1978). However, a pup can already do something by itself to compensate, in part, for the effect of hypothermia on blood circulation. Like many other animals, to help maintain cardiac output during physiologically stressful situations like extreme cooling, rat pups can perform a physiological manoeuvre known as the abdominal compression reaction (ACR) (Youmans et al. 1974). During an ACR, the abdominal muscles of the pup are contracted while the larynx is used as a brake during expiration, producing an increased intra-abdominal pressure which propels peripheral venous blood back to the heart.

On the basis of a series of experiments, Blumberg and his colleagues concluded that the infant rat's ultrasounds that influence the mother's behaviour are actually the incidental byproduct of these rapid abdominal contractions while the larynx is closed during expiration: a physiological process that serves to maintain cardiovascular activity in response to extreme cooling (Blumberg \& Sokoloff, 2001; Blumberg, 2009). If so, there is no evidence that the UVSs of infant rats are an adaptation to influence the mother's behaviour 6 .

It is important to point out that despite behavioural, physiological, pharmacological and neurological evidence in its favour (see Schwarting \& Wöhr 2012), the by-product hypothesis is not yet universally accepted (Shair \& Jasper, 2003; Arch \& Narins 2008). However, this case has several important implications for how we understand communication. ${ }^{7}$

According to the influence approach, in order to demonstrate that something is a signal and not a cue, it is necessary to show that it evolved owing to the response it elicits (cf. Diggle et al., 2007: 1242). So, if there is evidence that pups' USVs have not evolved owing to the response they elicit, but they are just an incidental acoustic by-product of a physiological response to hypothermia, then we should conclude that pups' USVs are cues and not signals, and consequently, that USVs-mediated infant-mother interactions are not communication. To be considered a signal, a trait $\mathrm{T}$ (such as USVs) is required to have

\footnotetext{
${ }^{6}$ Yet, although pups have not been selected for emitting USVs, the mother response to USVs may be an adaptation. While pups are deaf to their own USVs, the mother's hearing curve is tuned specifically to the pup's vocalisation frequency, and several playback studies have shown that USVs alone are able to elicit searching behaviour by mothers (Blumberg \& Alberts, 1990).

${ }^{7}$ Blumberg \& Alberts (1997) discuss more examples of signals that are considered incidental by-products of diverse aspects of organisms.
} 
propagated within a population in virtue of this effect (positive natural selection) and/or to have been maintained or retained against mutants which lacked $\mathrm{T}$ (negative natural selection or purifying selection). If (1) we assume the hypothesis of USVs as by-product of the abdominal compression reaction (ACR) as valid, and (2) we take into account that ACR can be found in several mammal taxa and that it is an ancestral trait in rodents (plesiomorphy), we can discard the possibility that the operation of ACR propagated in virtue of its influence upon maternal behaviour. Given the absence of evidence supporting the hypothesis that the production of USVs had been retained through purifying selection, the etiological approach does not appear to be able to account for USVs as signals.

The problem with the etiological approach to communication as influence is that if we accept the hypothesis of USVs as by-products, then this approach does not characterise them as signals. However, so far as we know, no scientist has proposed removing these infant-mother interactions form the category of communication. On the contrary, scientists involved in this discussion agree on the fact that being an incidental non-selected byproduct does not preclude us from continuing thinking about USVs as signals mediating communicative interactions between pup and mothers ${ }^{8}$. This view is shared by Drew Rendall, an advocate of the influence approach to biological communication. Agreeing on the fact that "the calls are an incidental by-product of a physiological response to cold stress for which selection has favoured maternal perceptual sensitivity and behavioural responsiveness" (Rendall \& Vasey 2002: 637), nevertheless Rendall still describes this case as "an example of functionally integrated communication between mothers and pups" (2002: 637).

We concluded the previous section by asking whether it is conceivable for scientists who work on biological communication to characterise a certain influencing interaction as a signal even if it is not an adaptation, and how they would actually deal with a case like that. The case of the acoustic by-product hypothesis examined in this section shows how it is

\footnotetext{
${ }^{8}$ See for example, Blumberg et al. (2000: 81): "It is important to emphasize that incidental emission of the vocalization does not preclude the development of a communicatory relationship between infant and mother". According to Arch \& Narins (2008: 1024) "if an ultrasonic utterance is emitted for a function other than intraspecific communication, or as an incidental byproduct of a physiological process or biomechanical strain, it cannot be said to be adapted for communication. However, if the ultrasounds have a consistent influence on conspecific behavior, then the vocalization may be considered exapted for communication". Gagliano (2013: 792) specifies that this case can be considered as an instance of communication regardless of the fact that the emission of the signal by the pup is not an adaptation, while the response of the mother is: " [...] the acoustic emissions seem to be just the mere and simple by-product of physiological and biomechanical strain. Yet, the incidental nature of ultrasonic emissions does not preclude the evolutionary development of a communicatory relationship between individuals. [...] In the rodent example, the ultrasonic vocalizations of a pup being cooled outside the nest reliably elicit a phonotaxic response in the mother (Ehret and Haack 1984). Hence, regardless of the proximate cause of the signal's emission, these ultrasounds trigger a behavior in the mother that is beneficial to the signaling pup (Blumberg and Alberts 1997). Clearly, this is a signal that transfers some information to the receiver, whose behavioral activities have changed in an adaptive way, hence increasing the genetic fitness of the pup-mother system".
} 
indeed possible, at least in principle, to conceive of cases of communication in which signals are not the products of adaptations. It constitutes a case where the clear possibility of a trait not being an adaptation does not preclude scientists from continuing to think about it as a signal, and about the phenomenon in question as communication. They address it as a case of communication on the basis of its current features, without the need to provide a justification in terms of the history of the signal trait.

This is difficult to accommodate within the influence approach. If pups' USVs are just an incidental acoustic by-product, and they have not been naturally selected in virtue of triggering maternal retrieving responses, then, according to the influence approach, they are not signals. Conversely, if we can think about USVs as being at the same time both signals which mediate communication between mothers and pups, and incidental non-selected consequences of a physiological process, then something is wrong with the influence approach, at least as it has been developed so far.

\subsection{The synthetic modelling of biological communication}

The example of USVs in rat pups shows an inconsistency in the influence approach. When biologists characterise an interaction as communicative, they may do it on the basis of things happening at the present time, regardless of how things were in the remote evolutionary past. This is at odds with the influence approach to communication. The problem is even more evident if we consider it from the point of view of synthetic biology. A thriving line of research in this field focuses on the interactions between artificial cells (protocells), synthetically modified cells, and natural cells, with different practical and theoretical goals. One approach within this line aims specifically at the realisation of synthetic models of biological communication. It consists in designing sensory-effector biomolecular mechanisms that allow protocells to be receptive to signals emitted by biological cells (and vice versa), and to change their behaviour accordingly, for example by producing specific metabolites and releasing them into the environment (Gardner et al. 2009; Rampioni et al., 2014; Lentini et al., 2017).

Let us consider a case in which protocells (the receivers) are designed to contain biomolecular mechanisms such as riboswitches ${ }^{9}$, that make them sensitive to stress signal molecules released by natural cells (the senders). The presence of such signal molecules in the protocells activates the synthesis of proteins and metabolites, that in turn can be supplemented to the sender cells, which need them for their functioning. This case seems to be a clear instance of communication where sensory-effector functional loops are

\footnotetext{
${ }^{9}$ Riboswitches are regulatory RNA strands located in untranslated regions of larger mRNA molecules. They can bind small molecules (ligands) and control gene expression at the transcriptional or translational level. They can be used to design protocells capable to sense small molecules and modulate their internal synthetic mechanisms of gene expression accordingly (see Martini \& Mansy, 2011).
} 
established between natural cells (senders) and protocells (receivers) ${ }^{10}$. However, it cannot be categorised as a case of communication according to the influence approach, despite the fact that an influence relationship takes place between natural cells and protocells. The influence account as based on etiological functions, in fact, a priori rules out the very possibility of artificial, non-evolved communication systems, such as those realised in these cases $^{11}$. This either makes the influence approach useless in the context of synthetic biology, or it excludes this whole research field from the study of communication. ${ }^{12}$

In this section we discussed three problems exhibited by the influence approach: the mismatch between theory and practice, the conflation of an operational characterisation with an origin explanation, and the problem of synthetic realisations of biological communication. Their roots lie in the underlying etiological account of biological functions, according to which the function of a trait is to produce the effects for which past occurrences of that trait were naturally selected. When confronting these issues, advocates of the influence account to communication are faced with two options. The first is to bite the bullet and accept the limits of the approach: i.e. a discordance between the theory of communication and a considerable part of scientific practice, with the removal from the domain of communication of phenomena which are currently studied by biologists as instances of communicative interactions. Moreover, it implies excluding from the study of communication a whole field of research in synthetic biology, which is playing an increasingly important role in this discipline, and which is in need of theoretical tools.

The second option is to revise the influence approach by adopting a different account of functions. In the next section we explore this possibility by critically rethinking the influence approach based on the organisational account of biological functions. We show how, by characterising functions in terms of contribution to the maintenance of the current organisation of a biological system, this alternative approach makes it possible to overcome the limits of the influence account while keeping its virtues.

\footnotetext{
${ }^{10}$ See Bich \& Frick (2018) for a more detailed discussion of synthetic models of biological communication.

${ }^{11}$ The fact that synthetic communication systems lack functions in an evolutionary sense, does not necessarily mean that they do not exhibit functions at all. They may have functions extrinsically established by a designer, but they may also exhibit functions that can be identified intrinsically through criteria that are not based on evolution, as it will be discussed in Section 4. The latter case is particularly relevant to the discussion of biological communication in the context of synthetic biology. This type of functions allows characterising an interaction in the same way as for non-artificial biological systems: as a phenomenon of communication for the systems involved in the interaction, rather than for an external user or designer.

${ }^{12}$ One might object that, in addition to natural selection, processes of artificial selection might also give rise to evolved synthetic communication systems to which functions could be attributed in terms of evolutionary adaptations. Yet, as shown in the example, the work on communication carried out in synthetic biology shows that this phenomenon can be produced without the need to generate it through artificial evolution and, therefore, that the latter is not a necessary condition for the realisation and study of communication systems.
} 


\section{An organisational approach to biological communication}

According to the influence approach to communication, a trait of an organism can be identified as a signal only if it has the function of influencing another organism. An etiological theory of functions, where this functional requirement can be stated in terms of adaptations and natural selection, has been usually taken for granted so that the influence approach is sometimes called "the adaptationist approach" (Scott-Philipps 2008). However, etiological theories are not the only ones available. Given that it is possible to think about biological functions in different ways, strictly speaking the adaptationist approach to communication is just a particular version of the influence approach. As an alternative, we propose an influence approach to communication based on the organisational theory of biological functions.

\subsection{The organisational approach to biological functions}

Inspired by the pioneering work of theoretical biologists such as Piaget (1967), Rosen (1972), Pattee (1973), Maturana and Varela ([1973]1980), among others, the organisational framework puts a major emphasis on the fact that a biological system is a self-maintaining system. Several formulations of organisational theories of functions have been proposed in the last two decades, among others by Schlosser (1998), Collier (2000), Bickhard (2000; 2004), McLaughlin (2001), Christensen \& Bickhard (2002), Delancey (2006), Edin (2008), Mossio et al. (2009). The common aspect to all these accounts is that they ground functional attributions in the contributions of traits to the maintenance of the system that harbours them.

In developing our proposal for an organisational-influence approach to communication, we follow the account developed by Matteo Mossio, Cristian Saborido and Alvaro Moreno (Mossio et al. 2009; Saborido et al. 2011). In this view, a biological system essentially realises a distinctive regime of causation able to produce and maintain the parts that contribute to its operations, and to promote the conditions of its own existence through its interaction with the environment. This specific causal regime of self-maintenance traditionally known as organisational closure (Piaget, 1967; Varela, 1979) - is realised by a set of internally produced structures acting as constraints which harness biochemical and biomolecular processes. These internally produced constraints are organised in such a way that they are mutually dependent for their production and maintenance, and collectively contribute to maintain the conditions at which the whole network can persist (Montévil \& Mossio, 2015; Moreno \& Mossio, 2015; Winning \& Bechtel, 2018). They do so by controlling the underlying flow of energy and matter in such a way as to maintain the system in far from equilibrium conditions.

The notion of closure characterised in terms of constraints focuses on the distinctive capability of living systems to contribute to their own conditions of existence and to those 
of their parts (Mossio \& Bich, 2017). This causal regime explains the existence of a component by referring to its effects, and it grounds functional attributions. Within a selfmaintaining system subject to closure, functions are interpreted as specific causal effects of a part or trait, which - acting as a constraint - contributes to the maintenance of the organisation and therefore, ultimately, of the part itself (Mossio et al., 2009).

As formulated by Saborido et al. $(2011)^{13}$, the organisational theory states that a trait $\mathrm{T}$ has a function $\mathrm{F}$ within the organisation $\mathrm{O}$ of a system $\mathrm{A}$ if and only if:

C1) $\mathrm{T}$ contributes to the maintenance of the organisation $\mathrm{O}$ of $\mathrm{A}$;

C2) $\mathrm{T}$ is produced and maintained under some constraints exerted by $\mathrm{O}$;

C3) A realises organisational closure.

Let us think about the classical example of the function of the heart. This definition implies that as a component of a biological self-maintaining system realising closure, the heart has the function of pumping blood since (C1) pumping blood contributes to the maintenance of the organism by allowing blood to circulate, which in turn enables the transport of nutrients to and waste away from cells, the stabilisation of body temperature and $\mathrm{pH}$, and so on. At the same time, $(\mathrm{C} 2)$ the heart is produced and maintained under various constraints exerted by the organism, whose overall integrity is required for the ongoing existence of the heart itself. Lastly (C3), the organism realises organisational closure since it is constituted by a set of mutually dependent structures each of them making different yet complementary contributions to the self-maintenance of the system.

\subsection{An organisational account of biological communication: reframing the influence approach}

In its most general form, the influence approach to communication minimally implies a receiver responding to a sender's signal, where a signal is some trait of the sender (i) whose presence triggers some response in the receiver, and (ii) which has the function of triggering such responses. By adopting the organisational approach to functions, the influence account of communication can be reformulated in such a way that to say that a signal is functional specifically means that it contributes to the maintenance of the current organisation of the sender, without necessarily appealing to its evolutionary history. In this view, communication in the most basic sense implies that (i) a receiver responds to a signal emitted by the sender, and (ii) that a signal is a sender's trait that by triggering some response in a receiver, contributes to maintain the organisation that, in turn, is responsible to produce and maintain the signal trait itself.

\footnotetext{
${ }^{13}$ See also Mossio et al. (2009).
} 
More formally, we can outline a (minimal, sender-centred version of the) organisational-influence approach to communication, according to which:

A system A communicates with a system B by means of a trait $\mathrm{T}$ acting as a signal $\mathrm{S}$ within a self-maintaining organisation $\mathrm{O}$, if an only if:

S1) a trait $\mathrm{T}$ of system A triggers some response $\mathrm{R}$ in system $\mathrm{B}$;

S2) $\quad T$ is produced under some constraints exerted by the self-maintaining organisation $\mathrm{O}$ realizing closure;

S3) the response $\mathrm{R}$ triggered by $\mathrm{T}$ contributes to the maintenance of $\mathrm{O} .^{14}$

Operationally speaking, this account requires the interaction of two (or more) biological systems ${ }^{15}$, which realise regimes of organisational closure and are endowed with sensoryeffector regulatory mechanisms ${ }^{16}$. A process of communication is realised when the regulatory mechanisms of a system $\mathrm{A}$ (the sender) are activated by variations in the external or internal environments of A, and modulate its internal dynamics. The regulated system A, then, produces a signal $\mathrm{S}$, which triggers a regulatory action in a second system $\mathrm{B}$ (the receiver), which changes its own behaviour. The new behaviour of $\mathrm{B}$, in turn, is functional for the sender in the sense that it contributes to the maintenance of $\mathrm{A}$ in the context that activated the regulatory action of $\mathrm{A}$ in the first place.

Like the etiological-influence approach, this account can distinguish signals form cues, so that it characterises as instances of communication only a proper sub-set of all coordinated behavioural interactions between $\mathrm{A}$ and $\mathrm{B}$. The fact that an organisation $\mathrm{A}$ produces an effect $\mathrm{T}$ that elicits a response $\mathrm{R}$ in another organism $\mathrm{B}$, is a necessary but not a sufficient condition for communication in the biological sense. Communication (and saying that $\mathrm{T}$ is a proper signal and not a cue), requires that $\mathrm{T}$ has the biological function of triggering the response $\mathrm{R}$ in $\mathrm{B}$. More specifically, this implies that $\mathrm{i}$ ) $\mathrm{A}$ is a self-maintaining system, and ii) $\mathrm{R}$ (the receiver's response triggered by $\mathrm{T}$ ) is functional for $\mathrm{A}$, i.e. it contributes to the maintenance of $\mathrm{A}$.

\footnotetext{
${ }^{14}$ We have presented a sender-centred version just for the sake of simplicity. However, it is easy to go from this sender-centred version to a "full", cooperative version (see Sec. 2.2) of the organisational-influence approach to communication, by adding the additional clause S4: "the receiver's response is produced in a self-maintaining system realising closure; and it contributes to the maintenance of this system".

${ }^{15}$ By biological systems we refer here to systems that realise a regime of organisational closure and exhibit basic autonomy and agency, i.e. they are capable to self-maintain and to modify their environments in such a way as to contribute to their own existence (see Moreno \& Mossio, 2015 for more details). Not only fullfledged organisms are included in this category, but also for example the cells of a multicellular organism.

${ }^{16}$ According to the organisational framework, regulatory mechanisms are second-order functional constraints that modulate the activity of the basic first-order constitutive constraints in such a way as to maintain the system viable under internal or external variations (Bich et al. 2016). See Bich \& Moreno (2016) for a discussion of regulation in the context of sensory-effector mechanisms.
} 
Moreover, the organisational approach can also account for those cases of communication, like pup rats' USVs, that exhibit the same operational features as other communicative mechanisms and are recognised as such by biologist, but cannot be explained by the etiological account. According to our proposal, whether USVs-mediated infant-mother interactions count as communication, does not need to be established in relation to the evolutionary origin and history of USVs. Rather, to decide whether a trait is a signal or not, we just need to observe the way in which this trait currently operates in the behavioural system in consideration, looking for the satisfaction of the three clauses contained in the organisational definition of signal introduced above.

Let us consider this case again from the operational point of view. A state of hypothermia in pups (the senders) activates a regulatory response aimed at compensating the effects of cold. It consists in modulating, by means of abdominal compression of the veins, the basic functional constraints involved in blood circulation. This operation has the effect of producing USVs (the signals), which trigger the sensory mechanisms in the mother (the receiver), and activate a regulatory response in her that produces a retrieval behaviour. This response is functional for the senders (the pups) in the context of hypothermia that activated the signalling mechanism in the first place, as the pups are returned to the warmth of the nest. It is easy to see how these operations satisfy the organisational definition of signals: (S1) USVs' presence triggers some response in the receiver; (S2) USVs are emitted by pup rats, which are self-maintaining, organisationally closed systems; and (S3), the mother's response triggered by USVs contributes to the maintenance of the pups. So, unlike the standard etiological-influence approach, the new proposal is consistent with the stance adopted by biologists in considering pups USVs as signals and USVs-mediated infant-mother interactions as a case of communication, without the need to appeal to evolutionary history.

This approach does not only discriminate between signals and cues. Within the organisational framework itself, communication can be distinguished from other types of functional interactions biological systems can undergo in different contexts and through different means while realising closure. For example, communication by means of molecular signalling is different from the metabolic exchanges that characterise many symbiotic interactions ${ }^{17}$. Whereas a signal molecule plays its role as signal in virtue of its capability to trigger a regulatory action in the sensory-effector mechanisms of the receiver - and ultimately give rise to a functional influence loop - a metabolite is used as a substrate (it is consumed) in the metabolic processes of the receiver. Metabolic interactions do not imply a functional influence by the sender upon the receiver. They realise functional

\footnotetext{
${ }^{17}$ A typical example is the exchange of amino acids and metabolites between hosts and endosymbionts, such as in insect cells (see for example Wu et al. 2006).
} 
couplings through the exchange of metabolites that are released in the environment and used in the metabolism of the systems involved in the interaction.

Moreover, communication can be involved in, but does not coincide with, ecological interactions. In the organisational framework, ecological interactions between organisms can realise a form of collective closure between organisms, whose self-maintaining regime goes beyond the individual organisms. This interspecies collective regime of organisational closure is realised by means of mutual constraints exerted by (groups of) organisms on one another's external boundary conditions, and it can ground functional attributions in terms of contribution to the more comprehensive ecological organisation (Nunes-Neto et al., 2014). The difference with communication lies in the fact that sender's signals do not constrain the external boundary conditions of the receiver, but they directly trigger its sensory-effector mechanisms. Therefore, the functional loop realised by communication as influence alone does not constitute per se a regime of closure at the ecological level. Yet, communicative interactions in some cases may be involved in a more encompassing collective organisation subject to closure at the level of communities or ecosystems. They can do so by modulating the way the receiver's behaviour contributes to the maintenance of such a wider regime of closure in which signals are produced ${ }^{18}$.

\subsection{Potential objections on reproductive and altruistic signals: signals whose effects in the receiver do not contribute to the maintenance of sender sensu stricto}

The organisational-influence approach is capable to account for cases, such as pups' USVs, in which the sender's signal triggers in the receiver responses that directly contribute to the maintenance of the sender. However, there are some obvious cases of communication that at first glance might not seem to fit into this schema. Male crickets, for instance, raise their forewings and rub them together producing chirps and trills, and female crickets are attracted by these sounds. In this classical example of animal communication, the function of the male cricket's acoustic signals is to attract potential mates, and so they are commonly described as mate-attraction signals (Moiseff, 1978). Given that these obvious cases of signals contribute to the reproduction of the sender rather than its self-maintenance, one might object that they represent a problem to the organisational-influence approach to communication, as they do not appear to be subject to the kind of closure required. Thus, the new proposal would seem to fail to adequately account for mate-attraction and reproductive signals.

We must notice that reproductive signals represent a type of challenge to the organisational-influence approach to communication which is exactly analogous to the challenge faced by the organisational theory of biological functions in relation to reproductive traits in general. The sperm cell, for instance, is usually considered a

\footnotetext{
${ }^{18}$ See Section 4.3 below for more details on this point.
} 
functional trait (its function is fertilising the ovum), even though it does not seem to contribute to the maintenance of the same organisation which produces it. This concern was explicitly addressed by Saborido et al. (2011) and further elaborated by Mossio \& Saborido (2016), who argue that the organisational theory can indeed account for traits with reproductive functions. These traits contribute to the maintenance of systems which realise a self-maintaining organisation in the very same sense as that of systems whose parts are ascribed typical organismic functions. Given that the problem of reproductive signals can be seen as a particular case of the general problem of reproductive traits, the organisational-influence approach to communication can be defended using a similar strategy. So, let us review in more detail how the organisational theory can account for these traits.

Saborido et al. (2011) develop their solution to the problem of reproductive traits by pointing out four crucial points. First, a self-maintaining organisation occurs in time. Thus, ascribing functions to traits or parts requires taking into consideration a system that realises self-maintenance during a period of time long enough for organisational closure to be observed. Second, because of its dissipative nature, a biological system continuously undergoes changes and replacements of its material components but, despite these changes, it remains the same self-maintaining system over time. Third, the fact that a trait contributes to the maintenance of the organisation of a system at time $t 1$, and that a trait is produced and maintained under some constraints exerted by the organisation of the system in time $t 2$, can ground the ascription of functionality to the trait only if we assume that the organisation whose trait performs a function at time $t l$ is the same organisation that maintains the trait at $t 2$. Systems in $t 1$ and $t 2$ are temporal instances of the same encompassing self-maintaining organisation. Fourth, if a given system possesses a constitutive organisation because of its causal and material connection with a previous system possessing the same organisation, then both systems can be considered as temporal instances of the same encompassing system.

Given these premises, the solution proposed by Saborido et al. (2011) to the problem of reproductive traits is the following: a reproductive function is subject to organisational closure within the frame of a self-maintaining organisation whose extension in time goes beyond the lifespan of an individual organism. We can say that the sperm cell of an individual has the function of inseminating the ovum, because by inseminating the ovum the trait contributes to the replacement of the individuals that are part of an organisation, which in turn exerts several constraints under which the semen is produced and maintained in time (see also Moreno \& Mossio, 2015: 80).

Now we can return to the specific problem of reproductive signals. According to the organisational-influence approach to communication, a signal is a trait of a sender which, by triggering some response in a receiver, contributes to maintain an organisation that, in turn, contributes to produce and maintain the trait itself. Within this framework, we can say 
that male cricket's chirps and trills constitute signals and that their function is to attract females. Their effects, even if they do not contribute to the self-maintenance of the individual sender, do contribute to the maintenance of a temporally wider organisation, specifically by contributing to the production of a new organism to replace the previous one. This organisation (realised by both the reproducer and the reproduced system), in turn, exerts several constraints under which male crickets and their acoustic signals are produced and maintained. In sum, despite first appearances, the organisational-influence approach to communication can account for mate-attraction signals, courtship displays and reproductive signals in general, insofar as it can be shown that they do contribute to the maintenance of the same organisation that produces them and, thus, are subject to organisational closure.

A similar objection can arise from considering cases of altruistic communication, in which the signal emitted by the sender triggers in the receiver responses that contribute to the receiver's maintenance instead of the sender's, as should be the case for the influence approaches. When a ground squirrel sights a hawk, it emits a call, and the nearby squirrels respond to this call by hiding into their burrows. Traditionally, these alarm-calls have been interpreted as altruistic signals. Not only these signals do not contribute to the maintenance of the sender, but in some cases, by attracting the attention of predators to the sender, they can contribute to its own destruction (Maynard Smith, 1965; Hirth \& McCullogh, 1977). In this case we are again confronted with traits that are obvious instances of signals, even if it seems that they do not contribute to the maintenance of the same organisation that produces them, as instead required by the organisational-influence approach.

We can respond to this objection in a way that is not so dissimilar from the case of reproductive signals, i.e. by focusing on a wider system. Altruistic signals such as alarmcalls, can be said to contribute to the maintenance of an organisation whose selfmaintaining regime goes beyond the individual organisms. The new self-maintaining system, though, is not on a different, wider, time scale like in the case of reproductive signals. It is realised, instead, by a more extended collective organisation. As explained in Section 4.2, above organisms it is possible to identify encompassing socioecological systems or communities that realise their own specific regimes of self-maintenance and closure. Functions, at this level, are attributed according to contributions to this ecological organisation (see Nunes-Neto et al., 2014). Altruistic signals should be characterised as signals in the context of a more encompassing system - which includes both senders and receivers, among other subsystems - which realises a self-maintaining organisation, and this organisation exerts several constraints under which sender organisms and their respective alarm-calls can be produced and maintained. Consequently, like in the case of reproductive signals, the organisational-influence approach to communication can account for altruistic signals, insofar as it can be shown that they are subject to a regime of organisational closure. 


\section{Concluding remarks}

The occurrence of biological communication, or signalling, in the most basic sense implies a receiver responding to a signal produced by a sender. In its most general form, an influence approach to communication states that a signal is some trait of the sender such that its presence triggers some response in the receiver, and that it has the function of triggering such response. So far, virtually all promoters of the influence approach have adopted, in most of the cases uncritically, a particular theory of biological functions, namely, the etiological theory. Formulated in this way, the etiological-influence approach implies that all signals have been naturally selected in virtue of the responses they trigger in receivers. However, we have shown that if grounded in the etiological theory, the influence approach suffers from serious weaknesses.

Relying on a different account of functions, the organisational one, we have developed an alternative to the etiological-influence approach to communication, namely, the organisational-influence approach. According to the latter, a signal is a sender's trait which by triggering some response in a receiver, contributes to maintain an organisation that, in turn, contributes to produce and maintain the trait itself.

There are cases of communication in which the sender coincides with the organisation maintained through the signal's effect on the receiver. However, sometimes it may happen that the sender is just a proper part of the organisation maintained through the signal's effect on the receiver, as we saw in the case of reproductive and altruistic signals. The organisational-influence approach to communication can account for these cases of communication as far as it can be shown that the signals are subject to a regime of organisational closure.

The implications of an organisational-influence approach to biological communication are still to be explored and critically assessed. Yet, this account might open new directions in the search for a theoretical grounding of the notion of communication, within an alternative naturalistic perspective. Being based on the notion of influence, it is free of the potential problems surrounding the concept of information, central to the information approach to biological communication. In practical terms, it is also more parsimonious, insofar as it does not require to account for an exchange of information between sender and receiver. At the same time, it can easily account for communication between very different species such as for example between humans and their bacterial symbionts, as far as they realise a specific (functional) pattern of interactions. Relying on an organisational account of biological functions, it is free from the problems inherent to the etiological-influence approach. It can account for communicative phenomena that did not evolve as adaptations. These do not include only stable and well-established behaviours like pups' USVs, but also cases of sporadic or even unique interactions as far as they fit the definition. Moreover, the definition of communication in terms of organisation and influence can be employed in 
synthetic biology, where the objects of study are artificial systems, such as protocells or synthetically modified cells, which are not the product of evolution. The organisational notion of influence does not impose strong operational requirements for synthetic realisations. It only requires the design of sensory-effector mechanisms and the realisation of a certain pattern of interactions between organisms, according to which the response to a signal is functional for the sender. For these reasons it is not only applicable in, but particularly suitable also for an experimental field such as synthetic biology.

\section{Acknowledgements}

The authors thank Derek Skillings and Matteo Mossio for their careful reading and useful comments on a previous version of this paper. This work was funded by the Ministerio de Economia, Industria y Competitividad (MINECO), Spain ('Ramon y Cajal' Programme, RYC-2016-19798 for LB, and research project FFI2014-52173-P for AM and LB), by the Basque Government (Project: IT 590-13 for AM and LB) and by CONICYT, Chile (Doctoral Scholarship 21151293-CONICYT and Supporting Doctoral Thesis Scholarship for RF). AM acknowledges also a Salvador de Madariaga Fellowship PRX17/00379 and he thanks the IHPST (Paris) for hosting his research stay during the first semester of 2018.

\section{References}

Arch, V. S., \& Narins, P. M. (2008). "Silent” signals: Selective forces acting on ultrasonic communication systems in terrestrial vertebrates. Animal behaviour, 76(4), 1423.

Bich, L. \& Frick, R. (2018). Synthetic Modelling of Biological Communication: A Theoretical and Operational Framework for the Investigation of Minimal Life and Cognition. Complex Systems, 27(3), 267-287.

Bich, L. \& Moreno, A. (2016). The role of regulation in the origin and synthetic modelling of minimal cognition. BioSystems, 148, 12-21.

Bich, L. Mossio, M. Ruiz-Mirazo, K., \& Moreno, A. (2016). Biological regulation: controlling the system from within. Biology \& Philosophy, 31(2), 237-265.

Bickhard, M.H. (2000). Autonomy, Function, and Representation. Communication and Cognition, 17(3-4), 111-131.

Bickhard, M.H. (2004). Process and Emergence: Normative Function and Representation. Axiomathes, 14, 121-155

Blumberg, M. S. (2009). Body heat: temperature and life on earth. Cambridge MA: Harvard University Press.

Blumberg, M. S., \& Alberts, J. R. (1990). Ultrasonic vocalizations by rat pups in the cold: an acoustic by-product of laryngeal braking? Behavioral neuroscience, 104(5), 808. 
Blumberg, M.S. \& Alberts, J.R. (1997). Incidental emissions, fortuitous effects, and the origins of communication. In Owings DH, Beecher MD, Thompson NS (eds.), Perspectives in ethology. New York: Plenum Press (pp. 225-249).

Blumberg, M. S., \& Sokoloff, G. (2001). Do infant rats cry? Psychological review, 108(1), 83 .

Blumberg, M. S., Sokoloff, G., Kirby, R. F., \& Kent, K. J. (2000). Distress vocalizations in infant rats: what's all the fuss about? Psychological Science, 11(1), 78-81.

Bradbury, J. W., \& Vehrencamp, S. L. (2011). Principles of animal communication. 2th ed, Sunderland, MA: Sinauer Associates.

Branham, M.A., Wenzel, J.W. (2001). The evolution of bioluminescence in cantharoids (Coleoptera: Elateriodea). Florida Entomologist, 84, 565-586.

Cameron, A. M. (1966). Some aspects of the behaviour of the soldier crab, Mictyris longicarpus. Pacific Science 20 (2): 224-23.

Carazo, P., \& Font, E. (2010). Putting information back into biological communication. Journal of evolutionary biology, 23(4), 661-669.

Christensen, W. \& Bickhard, M. (2002). The process dynamics of normative function. The Monist, 85(1), 3-28.

Collier, J. (2000). Autonomy and process closure as the basis for functionality. Annals of the New York Academy of Science; 901:280-290.

Dawkins, R., \& Krebs, J. R. (1978). Animal signals: information or manipulation?,” In R. Krebs and N. B. Davies (Eds.), Behavioural Ecology: An Evolutionary Approach. Sutherland, MA: Sinauer Associates (pp.282-309).

De Ghett, V. J. (1978). The ontogeny of ultrasound production in rodents. In: Burghardt, GM; Bekoff, M. (eds.) The development of behavior: Comparative and evolutionary aspects. New York: Garland STPM Press (pp. 343-365).

Delancey, C. (2006). Ontology and teleofunctions: A defense and revision of the systematic account of teleological explanation. Synthese, 150, 69-98.

Di Paolo, E. A. (1997a). An investigation into the evolution of communication. Adaptive Behavior, 6(2), 285-324.

Di Paolo, E. A. (1997b). Social coordination and spatial organization: Steps towards the evolution of communication. In Fourth European Conference on Artificial Life. Cambridge MA: MIT Press (pp. 464-473).

Di Paolo, E. A. (1999). On the evolutionary and behavioral dynamics of social coordination: Models and theoretical aspects. Doctoral dissertation, University of Sussex. 
Diggle, S. P., Gardner, A., West, S. A., \& Griffin, A. S. (2007). Evolutionary theory of bacterial quorum sensing: when is a signal not a signal? Philosophical Transactions of the Royal Society of London B: Biological Sciences, 362(1483), 1241-1249.

Edin, B. B. (2008). Assigning biological functions: making sense of causal chains. Synthese, 161(2), 203-218.

Ehret, G., \& Haack, B. (1984). Motivation and arousal influence sound-induced maternal pup-retrieving behavior in lactating house mice. Zeitschrift für Tierpsychologie, 65(1), 2539.

Fögen, T. (2014). Animal Communication. In G. L. Campbell (Ed.), The Oxford Handbook of Animals in Classical Thought and Life, Oxford: Oxford University Press (pp. 216-232).

Gagliano, M. (2013). Green symphonies: a call for studies on acoustic communication in plants. Behavioral Ecology, 24 (4).

Gardner, P. M., Winzer, K., Davis, B. G. (2009). Sugar synthesis in a protocellular model leads to a cell signalling response in bacteria. Nature Chemistry, 1(5), 377-383.

Godfrey-Smith, P. (1994). A modern history theory of functions. Noûs, 28(3), 344-362.

Godfrey-Smith, P. (2013). Information and Influence in Sender-Receiver Models, with Applications to Animal Behavior. In U. Stegmann, (ed.), Animal Communication Theory: Information and Influence. Cambridge: Cambridge University Press (pp. 377-396).

Gould, S. J., \& Lewontin, R. C. (1979). The spandrels of San Marco and the Panglossian paradigm: a critique of the adaptationist programme. Proceedings of the Royal Society of London B: Biological Sciences, 205(1161), 581-598.

Hauser, M. D. (1996). The Evolution of Communication. Cambridge, MA: MIT Press.

Heil, M., \& Karban, R. (2010). Explaining evolution of plant communication by airborne signals. Trends in ecology \& evolution, 25(3), 137-144.

Hirth, D. H., \& McCullough, D. R. (1977). Evolution of alarm signals in ungulates with special reference to white-tailed deer. The American Naturalist, 111(977), 31-42.

Kalkman, D. (2017). New problems for defining animal communication in informational terms. Synthese, DOI: 10.1007/s11229-017-1598-2.

Kauffman, S. (2000). Investigations. Oxford: Oxford University Press.

Keely, S. J. (2017). Decoding host-microbiota communication in the gut - now we're flying! The Journal of Physiology, 595(2), 417-418.

Keller, L., \& Surette, M. G. (2006). Communication in bacteria: an ecological and evolutionary perspective. Nature Reviews Microbiology, 4(4), 249-258. 
Krebs, J.R., \& Dawkins, R. (1984). Animal signals: Mind reading and manipulation . In J. R. Krebs \& N. B. Davies (Eds.), Behavioral Ecology: An Evolutionary Approach (Second edition), pp. 380-402. Oxford: Blackwell.

Laidre, M. E., \& Johnstone, R. A. (2013). Animal signals. Current Biology, 23(18), R829R833.

Lentini, R., Martín, N. Y., Forlin, M., Belmonte, L., Fontana, J., Cornella, M., et al. (2017). Two-Way Chemical Communication between Artificial and Natural Cells. ACS Central Science, 3(2), 117-123.

Lewis, D. K. (1969). Convention: A Philosophical Study. Cambridge, MA: Harvard University Press.

Lloyd, E. A. (2015). Adaptationism and the logic of research questions: how to think clearly about evolutionary causes. Biological Theory, 10(4), 343-362.

Lloyd, J. (1966). Studies on the flash communication system in Photinus fireflies. Ann Arbor: Museum of Zoology of University of Michigan

Martinez, M. \& Godfrey-Smith, P. (2016). Common Interest and Signaling Games: A Dynamic Analysis. Philosophy of Science, 83, 371-392.

Martini, L. \& Mansy, S. (2011). Cell-like systems with riboswitch controlled gene expression. Chemical communications, 47(38), 10734-10736.

Maturana, H. \& Varela, F. J. ([1973]1980). Autopoiesis and Cognition. The Realization of the Living. Dordrecht: Reidel Publishing.

Maynard Smith, J. (1965). The Evolution of Alarm Calls. The American Naturalist, 99(904), 59-63.

Maynard Smith, J. \& Harper, D.G.C. (2003). Animal Signals. Oxford: Oxford University Press.

McLaughlin, P. (2001). What Functions Explain. Functional Explanation and SelfReproducing Systems. Cambridge: Cambridge University Press.

Millikan, R. G. (1989). In defense of proper functions. Philosophy of science, 56(2), 288302.

Moiseff, A., Pollack, G. S., \& Hoy, R. R. (1978). Steering responses of flying crickets to sound and ultrasound: mate attraction and predator avoidance. Proceedings of the National Academy of Sciences, 75(8), 4052-4056.

Montévil M., \& Mossio M. (2015). Biological organisation as closure of constraints. Journal of Theoretical Biology, 372, 179-191. 
Moreno, A., Mossio, M. (2015) Biological autonomy: a philosophical and theoretical enquiry. New York: Springer.

Morton, E. S. (2017). Animal Vocal Communication: Assessment and Management Roles, 2th ed. Cambridge: Cambridge University Press.

Mossio, M., \& Bich, L. (2017). What makes biological organisation teleological? Synthese, 194(4), 1089-1114

Mossio, M., \& Saborido, C. (2016). Functions, Organization and Etiology: A Reply to Artiga and Martinez. Acta Biotheoretica, 64(3), 263-275.

Mossio, M., Saborido, C. \& Moreno, A. (2009). An Organizational Account of Biological Functions. British Journal of Philosophy of Science, 60(4), 813-841.

Neander, K. (1991). The teleological notion of 'function'. Australasian Journal of Philosophy, 69(4), 454-468.

Nunes-Neto N., Moreno A., \& El-Hani C. N. (2014). Function in ecology: An organizational approach. Biology \& Philosophy, 29(1), 123-141

Otte, D. (1974). Effects and functions in the evolution of signaling systems. Annual Review of Ecology and Systematics, 5, 385-417.

Owings, D. H., \& Morton, E. S. (1998). Animal vocal communication: a new approach. Cambridge: Cambridge University Press.

Owren, M. J., Rendall, D., \& Ryan, M. J. (2010). Redefining animal signaling: influence versus information in communication. Biology \& Philosophy, 25(5), 755-780.

Page, R. A., \& Ryan, M. J. (2005). Flexibility in assessment of prey cues: frog-eating bats and frog calls. Proceedings of the Royal Society B, 272, 841-847.

Pattee, H. H. (1973). The Physical Basis and Origin of Hierarchical Control. In H. H. Pattee (ed.), Hierarchy Theory, New York: Braziller (pp. 73-108).

Piaget, J. (1967). Biologie et Connaissance. Paris: Gallimard.

Pigliucci, M., \& Kaplan, J. (2000). The fall and rise of Dr Pangloss: adaptationism and the Spandrels paper 20 years later. Trends in ecology \& evolution, 15(2), 66-70.

Rampioni, G., Mavelli, F., Damiano, L., D’Angelo, F., Messina, M., Leoni, L., Stano, P. (2014). A synthetic biology approach to bio-chem-ICT: first moves towards chemical communication between synthetic and natural cells. Natural Computing, 13, 333-349.

Rendall, D., \& Owren, M. J. (2013). Communication without meaning or information: abandoning language-based and informational constructs in animal communication theory. In H. Stegmann, Animal communication theory: information and influence, Cambridge: Cambridge University Press (pp. 151-182). 
Rendall, D., \& Vasey, P. (2002). Metaphor muddles in communication theory. Behavioral and Brain Sciences, 25(5), 637-637.

Rendall, D., Owren, M. J., \& Ryan, M. J. (2009). What do animal signals mean? Animal Behaviour, 78(2), 233-240.

Rhebergen F., Taylor R. C., Ryan M. J., Page R. A., \& Halfwerk W. (2015) Multimodal cues improve prey localization under complex environmental conditions. Proceedings of the Royal Society B, 282: 20151403.

Rosen R. (1972). Some relational cell models: The metabolism-repair systems. In R. Rosen (Ed.), Foundations of Mathematical Biology. Volume II Cellular Systems. Academic Press: New York (pp. 217-253).

Rosen, R. (1991). Life Itself. New York: Columbia University Press.

Saborido, C., Mossio, M., \& Moreno, A. (2011). Biological organization and crossgeneration functions. The British Journal for the Philosophy of Science, 62(3), 583-606.

Salmon, W. C. (1998). Causality and explanation. Oxford: Oxford University Press.

Scarantino, A. (2010). Animal communication between information and influence. Animal Behaviour, 79(6), e1-e5.

Scarantino, A. (2013). Animal communication as information-mediated influence. In H. Stegmann (Ed.), Animal communication theory: information and influence, Cambridge: Cambridge University Press (pp. 63-81).

Scattoni, M. L., Crawley, J., \& Ricceri, L. (2009). Ultrasonic vocalizations: a tool for behavioural phenotyping of mouse models of neurodevelopmental disorders. Neuroscience \& Biobehavioral Reviews, 33(4), 508-515.

Schaefer, H. M., \& Ruxton, G. D. (2009). Deception in plants: mimicry or perceptual exploitation? Trends in Ecology \& Evolution, 24(12), 676-685;

Schiestl, F. P., Peakall, R., Mant, J. G., Ibarra, F., Schulz, C., Franke, S., \& Francke, W. (2003). The chemistry of sexual deception in an orchid-wasp pollination system. Science, 302(5644), 437-438.

Schlosser, G. (1998). Self-re-production and functionality: A systems theoretical approach to teleological explanation. Synthese, 116, 303-354.

Schwarting, R. K. W., \& Wöhr, M. (2012). On the relationships between ultrasonic calling and anxiety-related behavior in rats. Brazilian Journal of Medical and Biological Research, 45(4), 337-348.

Scott-Phillips, T. C. (2008). Defining biological communication. Journal of evolutionary biology, 21(2), 387-395. 
Scott-Phillips, T. C. (2009). The quest for a general account of communication: A review of Sociobiology of Communication. Journal of Evolutionary Psychology, 7(3), 245-249.

Seyfarth, R. M., Cheney, D. L., \& Marler, P. (1980a). Monkey responses to three different alarm calls: evidence of predator classification and semantic communication. Science, 210(4471), 801-803.

Seyfarth, R. M., Cheney, D. L., \& Marler, P. (1980b). Vervet Monkey Alarm Calls: Semantic Communication in a Free-Ranging Primate. Animal Behaviour, 28, 1070-1094.

Seyfarth, R. M., Cheney, D. L., Bergman, T., Fischer, J., Zuberbühler, K., \& Hammerschmidt, K. (2010). The central importance of information in studies of animal communication. Animal Behaviour, 80(1), 3-8.

Shair, H. N., \& Jasper, A. (2003). Decreased venous return is neither sufficient nor necessary to elicit ultrasonic vocalisations of infant rat pups. Behavioral neuroscience, 117(4), 840.

Silverman, J. L., Yang, M., Lord, C., \& Crawley, J. N. (2010). Behavioural phenotyping assays for mouse models of autism. Nature Reviews Neuroscience, 11(7), 490-502.

Stegmann, U. E. (2009). A consumer-based teleosemantics for animal signals. Philosophy of Science, 76(5), 864-875.

Varela, F. (1979). Principles of Biological Autonomy. Dordrecht: North Holland.

Waters, C. M., \& Bassler, B. L. (2005). Quorum sensing: cell-to-cell communication in bacteria. Annual Review of Cell and Developmental Biology., 21, 319-346.

Williams, G.C. (1966). Adaptation and Natural Selection. Princeton: Princeton University Press.

Wilson, E. O. (1975). Sociobiology: the new synthesis. Cambridge, MA: Belknap.

Winning, J. \& Bechtel, B. (2018). Rethinking Causality in Biological and Neural Mechanisms: Constraints and Control. Minds and Machines, doi: 10.1007/s11023-0189458-5.

Wu D., Daugherty S.C., Van Aken S.E., Pai G.H, Watkins K.L., et al. (2006) Metabolic complementarity and genomics of the dual bacterial symbiosis of sharpshooters. PLoS Biology, 4(6): e188.

Youmans, W. B., Tjioe, D. T., \& Tong, E. Y. (1974). Control of involuntary activity of abdominal muscles. American Journal of Physical Medicine \& Rehabilitation, 53(2), 5774. 